\title{
Is Energy Storage an Economic Opportunity for the Eco-Neighborhood?*
}

\author{
Hélène Le Cadre \\ MINES ParisTech, \\ Centre for Applied Mathematics, \\ Sophia Antipolis, France \\ helene.le_cadre@mines-paristech.fr \\ David Mercier \\ CEA, LIST, \\ 91191 Gif-sur-Yvette Cedex, France \\ david.mercier@cea.fr
}

\begin{abstract}
In this article, we consider houses belonging to an eco-neighborhood in which inhabitants have the capacity to optimize dynamically the energy demand and the energy storage level so as to maximize their utility. The inhabitants' preferences are characterized by their sensitivity toward comfort versus price, the optimal expected temperature in the house, thermal loss and heating efficiency of their house. At his level, the eco-neighborhood manager shares the resource produced by the eco-neighborhood according to two schemes: an equal allocation between the houses and a priority based one. The problem is modeled as a stochastic game and solved using stochastic dynamic programming. We simulate the energy consumption of the eco-neighborhood under various pricing mechanisms: flat rate, peak and off-peak hour, blue/white/red day, peak day clearing and a dynamic update of the price based on the consumption of the eco-neighborhood. We observe that economic incentives for houses to store energy depend deeply on the implemented pricing mechanism and on the homogeneity in the houses' characteristics. Furthermore, when prices are based on the consumption of the eco-neighborhood, storage appears as a compensation for the errors made by the service provider in the prediction of the consumption of the eco-neighborhood.
\end{abstract}

Keywords: Eco-Neighborhood; Planning; Stochastic game theory; Energy storage; Pricing

\section{Introduction}

In the literature, the use of energy storage systems in houses has been presented as one of the main ways of saving energy in the smart grid [23]. Research in this area focused on designing new efficient low cost storage systems. The main economic argument that is raised to promote the use of storage in the smart grid is the following: if storage can

* The authors thank the three anonymous reviewers for their helpful comments and careful proofreading. 
be used to supply the house own consumption at peak electricity consumption times then it should be possible to lower peak demand such that fewer carbon intensive and expensive plant generators are required. As a result, from an ecological point of view, it would contribute to reducing both energy costs and carbon emissions. Furthermore, storage can be used to compensate for the variability of renewable energy generation. In practice, such energy storage may take the form of electric vehicles equipped with lithium-ion batteries or plug-in hybrid electric vehicles] according to Voice et al. [23]. Other systems of storage based on hydrogen or methanation exist. But, they do not seem to be the majority.

For such storage systems to be viable, consumers should have clear incentives to store energy because they are at the heart of the system. The design of such incentives requires the understanding of the consumers ${ }^{2}$ preferences in terms of sensitivity toward price, comfort, of the impact of the heterogeneity in the house characteristics in terms of thermal loss and heating efficiency, of priority schemes and pricing mechanisms. Thermal loss and heating efficiency will be used throughout the article to parametrize the houses. We recall briefly their definition:

- Thermal losses are heat losses, or losses of heat energy. One typical example is the idea of thermal loss through windows in a structure or through walls, and roof poor insulation.

- Heating efficiency is physically measured as the ratio of the heat transferred by the heating system to the heat content of the input source used to produce energy.

The aim of this article is to evaluate, using a game theoretic approach, the economic incentives for houses in an eco-neighborhood to store energy under two resource allocation schemes and various pricing mechanisms such as flat rate, peak and off-peak hour pricing, blue/white/red pricing, peak day clearing pricing and dynamic pricing. The term eco-neighborhood refers to the grouping within a territory of entities that will be generically called houses. The latter consume and produce mostly solar based energy [25]. When integrated into the smart grid, it will be called microgrids [8]. At the lower level the houses optimize independently their energy policy by adapting their consumption (demand and storage levels) whereas at the upper level a manager dynamically allocates the eco-neighborhood energy production between the houses to guarantee the viability of the ecosystem [25].

In many articles, game theory and multi-agent simulation are presented as the most appropriate approaches to tackle the multiple challenges associated to the smart grid operations. Saad et al. provided a complete review of the literature dealing with game theoretic methods for the smart grid [18]. The authors highlighted the fact that robust and smart demand side management is an essential characteristic of the smart grid systems. Indeed, to avoid service outages and blackouts which are unacceptable for the consumers, demand smoothing is traditionally performed through the implementation

\footnotetext{
${ }^{1}$ This perspective is sometimes referred to as vehicle to grid.

${ }^{2}$ Consumers can represent indifferently car drivers and/or house inhabitants.
} 
of demand side management techniques which are quite classical in the US [19]. Demand side management techniques include demand response approaches and energy efficiency. Demand response can be implemented in two ways: a first approach is to use dynamic pricing strategies; a second way is to use incentive based demand response where contracts are established between the transmission operator who manages the network, the consumers and other agents. In this article, we focus exclusively on price based demand response. In the literature, Maity and Rao proposed a game theoretic framework that enables the microgrids to decide on whether to store or use energy so as to meet the predicted demand of their consumers [11], [18]. The essence of the framework is based on two types of games: a non-cooperative solution for the Potluck problem which aims at reaching without communication an equilibrium where supply equals demand, and an auction game for determining the pricing in the microgrid network [11]. Mohsenian-Rad et al. devised a demand side management scheme that enables to schedule the shiftable home devices, while minimizing the overall energy consumption and thus, the charges on the consumers [12]. They proposed an algorithm which uses best response dynamics to find the Nash equilibrium while ensuring that no user has an incentive to cheat and announce an incorrect energy schedule. Voice et al. developed a framework to analyze agent-based micro-storage management for the smart grid [22], [23]. They designed a storage strategy with an adaptive mechanism based on predicted market prices for the consumers and empirically demonstrated that the average storage profile converges towards a Nash equilibrium. At that point, peak demands are reduced. Moreover, analyzing the social welfare at this equilibrium, they showed that it results in reduced costs. However, their model is restrictive in the sense that the consumers' preferences are based exclusively on the characteristics of their storage systems i.e., its maximum capacity, its efficiency and its running cost. Additionally, the consumers' utility coincides with budget minimization exclusively.

The main limitations of these articles are that few of them take into account the microgrid intrinsic characteristics and that all of them seem to ignore the consumers' preferences in terms of comfort versus budget, optimal temperature in the house, etc. Additionally, the uncertainty resulting from the generation of energy issued from renewable sources is modeled using density functions which shapes and parameters are adjusted a priory [16]. This last assumption seems rather unrealistic since it does not take into account the erratic nature of the renewable sources which rely on uncontrolable exogenous events. In [8], [9], we studied the double Stackelberg game which occurs between producers using either renewable or non renewable energies, the service providers and the aggregated consumers. The renewable energy production and the microgrid demand were represented by individual sequences, which involved no stochastic assumptions on the underlying processes.

Compared with [8], [9], we focus on a finer scale in this article, since we consider an individual microgrid and model the inter-relations between the houses composing the microgrid and the eco-neighborhood manager. There are two ways to fix the service provider's price per energy unit: either it is determined exogenously as the result of a predefinite pattern based on day/hour characteristics, or it is obtained as the output of the double Stackeberg game studied in [8], [9] once the consumers' demand has been 
aggregated in the microgrid.

The originality of this article lies in:

- The fact that we concentrate no more on load but on the comfort, which depends in turn, on the heat and on the internal temperature in the house. The simultaneous production of two different energies such as electricity and heat in the same process is called cogeneration. Heat storage measured through the comfort in the house, allows the monitoring of electric charge which produces electricity for specific needs. This choice of modeling can be justified by the fact that it is more easily measurable, taking a consumer's point of view, and that it is considered as the first criterion for the inhabitants to evaluate the environmental quality.

- The incorporation of the consumers' preferences through their profiles which contain the optimal temperature in their house, the house characteristics such as thermal loss and heating efficiency and the multi-criteria utility which takes into account the balance between the budget dedicated to heating and the comfort perceived by the inhabitants of the house.

- The introduction of a stochastic process to model the uncertainty associated to the renewable energy integration in the grid.

- The comparison of the economic incentives for the houses, to store energy, under two resource allocation schemes when various pricing mechanisms are used.

The article is organized as follows. In Section 2, we describe the model and the interplay between the agents. In Section 3 , the two-level game is solved analytically without storage capacity. In Section 4 , we consider that the houses have the capacity to store energy and we introduce uncertainty on the resource production from the econeighborhood through a Markov chain. The problem is then modeled as a finite horizon stochastic game and algorithms are proposed under two resource allocation schemes. Finally, simulations are performed in Section 5 to determine the impact of pricing mechanisms on the consumers' incentives to store energy in the eco-neighborhood.

\section{The model}

We consider $H \in \mathbb{N}^{*}$ houses belonging to the same eco-neighborhood managed by an eco-neighborhood manager $E$. $E$ produces energy by himself using solar panels, wind turbines, etc. The houses inside the eco-neighborhood need to gather enough energy to maximize their comfort while minimizing the budget dedicated to this provision. The involved players share hierarchical relations. We describe the economic relations between them:

- The service provider is in fact an energy provider. Both terms will be used interchangeably throughout the article. It collects energy from various energy sources (nuclear, coal, fuel, hydraulic, geothermal, solar, windpower, etc.) and 
distributes it to final users who can be individual consumers, firms, eco-neighborhoods, etc. Each house contracts only with one service provider and does not churn from one service provider to another during all the period of our study. This assumption holds well due to the local geographic aspect of the eco-neighborhoods. In this sense, the set made of provider and attached eco-neighborhood can be seen as an individual microgrid, as defined in [17].

- The eco-neighborhood manager $E$ allocates the energy resource that he has collected between the eco-neighborhood houses. In case of negative microgrid energy balance, he will also sell the missing quantities of energy to each house at a price $p(t)$ fixed by the service provider.

- In case of negative microgrid energy balance, the eco-neighborhood provider $E$ reattributes the revenue perceived for the missing quantities of energy to the service provider. To determine his unit price $p(t)$, the energy provider can choose between various pricing schemes that will be detailed in Section 5. flat rate, peak and off-peak hour, blue/white/red day, peak day clearing and dynamic pricing.

- Each of the $H$ individual houses receives energy and has the possibility to buy additional quantities from $E$. They can also store energy in batteries.

It is unrealistic to assume that there might be multiple eco-neighborhood managers competing at the eco-neighborhood level because in practice, it would require the end user to own a specific box to monitor energy consumption for each eco-neighborhood manager unless technological progress is made enabling the fusion of the consumptions on a single box. An additional problem is that this excess of technology might generate lassitude at the end user level. On the contrary, it is realistic to introduce competition between the energy producers delivering energy to the providers. The problem has been modeled as a market place in [8], [9] and solved using learning game theory.

It might be possible to model the interactions between the eco-neighborhood manager $E$ and the houses through a market place where the houses make independent bids to get energy access. Various forms of auctions or more generally mechanisms [5], [13], can be envisaged. The problem is that such an approach would require that each house be equipped with a sophisticated communication box and would inject large data flows in the smart grid. This last aspect would increase further the complexity of the communication layer of the smart grid which is already extremely complex due to the hierarchical and highly decentralized structure of the grid. As a result, it is more realistic to assume that the eco-neighborhood manager will act as a supervisor, planning the eco-neighborhood consumption and allocating its resource between the houses.

A house $h$ will be characterized by its profile: $\left(\theta_{h}^{0}, \kappa_{h}, \bar{\kappa}_{h}\right)$ where $\theta_{h}^{0}$ is the optimal temperature, $\kappa_{h}$ is the thermal loss and $\bar{\kappa}_{h}$, the heating efficiency in house $h$, for any $h=1, \ldots, H$. The aim of this article, schematized in Figure 1 , is to design a planning algorithm to allocate the resource produced by the eco-neighborhood between the houses depending on their profiles and on exogenous weather parameters 
so as to maximize the long-term expected discounted weighted social welfare of the eco-neighborhood that will be introduced in Subsection 2.1.

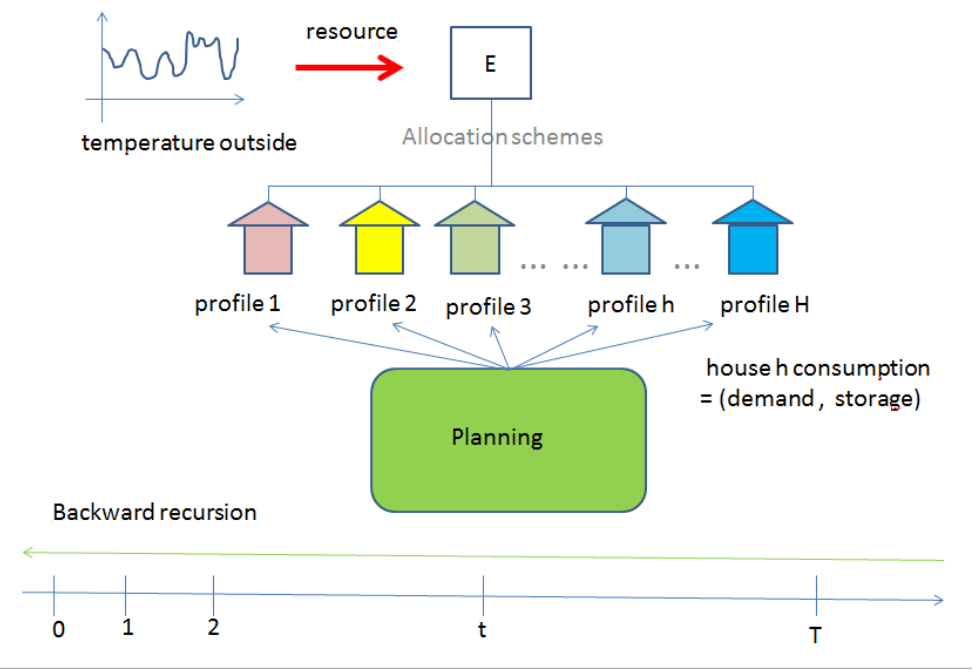

Figure 1: Planning the eco-neighborhood energy consumption.

\subsection{The agents}

In this subsection, we describe each agent's role, their interplay and give the formal expression of their utility.

House $h$ We denote as $Q_{h}(t)$ the stock level of house $h$ at time period $t$ and as $\Delta q_{h}(t)$ the variation of this stock between time periods $t-1$ and $t$. The maximum capacity of the battery is fixed at $Q_{\max } \in[0 ; 1]$. Therefore, $Q_{h}(t)$ belongs to the interval $\left[0 ; Q_{\max }\right]$. It is straightforward to define the stock level at time period $t$ as a function of the stock level at time period $t-1$ and of its variation: $Q_{h}(t)=Q_{h}(t-$ $1)+\Delta q_{h}(t)$ with the initialization $Q_{h}(0)=0$. Constraints on the stock variation are:

$$
\underbrace{-Q_{h}(t-1)}_{\text {maximum destocking }} \leq \Delta q_{h}(t) \leq \underbrace{Q_{\max }-Q_{h}(t-1)}_{\text {maximum stocking capacity }}
$$

For any $x \in \mathbb{R}$, we use the notation: $(x)_{+}=\max \{x ; 0\}$

At each time period $t$, house $h$ determines its energy demand $x_{h}(t) \in[0 ; 1]$. The energy demand of house $h$ can be satisfied:

(i) By drawing in the available stock $Q_{h}(t-1)$ 
(ii) By using the resource allocated by the eco-neighborhood manager $R_{h}(t)$

(iii) By buying the missing quantity $\left(x_{h}(t)-R_{h}(t)+\Delta q_{h}(t)\right)_{+}$from the econeighborhood manager $E$ who reattributes the perceived revenue to the service provider.

The utility of house $h$ is:

$$
\pi_{h}(t)=\lambda_{h} C_{h}\left(\theta_{h}(t)\right)-p(t)\left(x_{h}(t)-R_{h}(t)+\Delta q_{h}(t)\right)_{+}
$$

where $\theta_{h}(t)$ is the temperature in house $h$ and $\lambda_{h} \in \mathbb{R}$ is a parameter modeling the importance of the price with respect to the comfort level. A high value $\lambda_{h}$ (resp. a small value $\lambda_{h}$ ) means that house $h$ is more (resp. less) sensitive to comfort than to price. The comfort function of house $h, C_{h}(.) \in \mathcal{C}^{2}(\mathbb{R})$ is a continuous, twice differentiable function over $\mathbb{R}$ which admits continuous differentiates. It is supposed to be of Gaussian type centered in the optimal temperature $\theta_{h}^{0} \in \mathbb{R}_{+}$which can be customized for each house $h$ and normalized with a standard deviation of 13

$$
\mathcal{C}_{h}\left(\theta_{h}(t)\right)=\frac{1}{\sqrt{2 \pi}} \exp \left(-\frac{\left(\theta_{h}(t)-\theta_{h}^{0}\right)^{2}}{2}\right)
$$

The Gaussian assumption enables us to take into account threshold effects in the end user perception: comfort increases until a threshold is reached and then it decreases symmetrically. According to some studies performed at the end user level, the temperature is the first measure of comfort used by the end user. However, there exist other measures such as the quality of life, the minimization of the carbon footprint, the fact that the energy used thoughout the eco-neighborhood has been produced through green sources, etc. Such criteria have been omitted because they appear as secondary in a first approach.

For each house $h$, parameters $\lambda_{h}, \theta_{h}^{0}$ characterize the house perception of comfort and are not known a priori by the other houses. Identically, the home characteristics measured by its thermal loss and heating efficiency, are not known a priori by the other houses. Therefore, this is a game with incomplete information [13].

In Figure 2 (a), we have pictured the comfort function as a Gaussian density function centered around the optimal temperature of $\theta_{h}^{0}=21^{0} \mathrm{C}$ and of standard deviation 1. The standard deviation being normalized, it will be necessary to change the temperature scale by dividing all the possible values by $10^{\circ} \mathrm{C}$. As already mentioned in footnote, this change of scale will enable us to widen the range of values where the comfort function reaches high values around the optimal temperature.

The calibration of the comfort sensitivity parameter is not easy because it is used to adjust the comfort value and the budget dedicated to heating on a common scale

\footnotetext{
${ }^{3}$ The normalization of the standard deviation is introduced to simplify the choice of the parameters. To obtain realistic values for the comfort function and for the game outputs that will be defined in the next sections, it will be necessary to rescale the temperature range of values.
} 
so as to evaluate their respective influence in the house's utility. In Figure 2 (b), we have represented house $h$ 's utility $\pi_{h}($.$) as a function of comfort sensitivity parameter$ $\lambda_{h}$ and price $p($.$) while the temperature in the house is fixed at its optimal value i.e.,$ $\theta_{h}()=.\theta_{h}^{0}$ and there is one missing unit of energy i.e., $x_{h}()-.R_{h}()+.\Delta q_{h}()=$.1 . This setting coincides with a worst case because house $h$ needs to heat at its maximum to guarantee the reaching of the optimal temperature with no storage to compensate for its effort. In simulation experiments that will be described in Section 5 the price per energy unit will be supposed smaller than 60. As a result, according to Figure 2(b), it will be necessary to fix $\lambda_{h}$ greater than 150 to obtain a non-negative utility for house $h$.

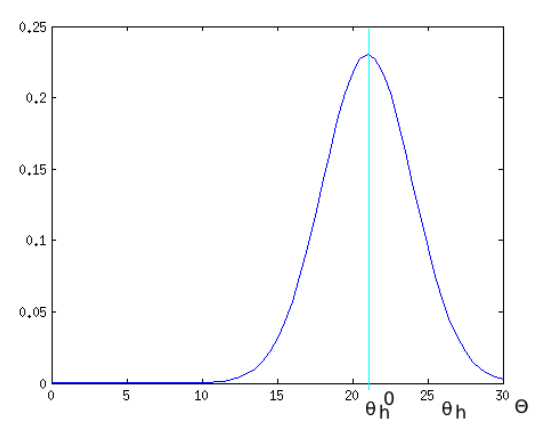

(a)

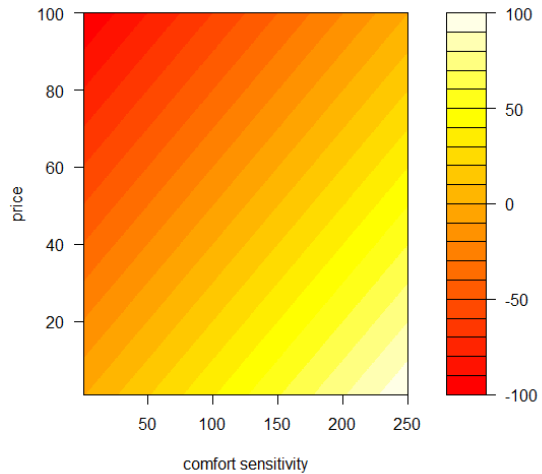

(b)

Figure 2: Calibration of the model parameters $\theta_{h}^{0}, \lambda_{h}$. In (a), the house $h$ 's comfort function is pictured as a gaussian density function centered around the optimal temperature for the house $\theta_{h}^{0}=21^{0} C$. In (b), house $h$ 's utility is represented as a function of comfort sensitivity parameter and price.

The eco-neighborhood manager $E$ The eco-neighborhood manager $E$ produces an energy resource $R(t) \in[0 ; 1]$ at time period $t$. He allocates it between the houses in such a way that: $R(t)=\sum_{h=1, \ldots, H} R_{h}(t)$ and $0 \leq R_{h}(t) \leq R(t), \forall h=1, \ldots, H$. As a non-lucrative agent, his utility is defined as the weigthed social welfare criterion: $\pi_{E}(t)=\sum_{h=1, \ldots, H} \alpha_{h} \pi_{h}(t)$. The coefficients $\alpha_{h}, \forall h=1, \ldots, H$ characterize the priority level between the houses. They are defined a priori and satisfy the following normalization constraints: $\sum_{h=1, \ldots, H} \alpha_{h}=1$ and $\alpha_{h} \geq 0, \forall h=1, \ldots, H$. In the rest of the article, we will consider the two following schemes:

- Scheme 1: All the houses have the same priority coefficient $\frac{1}{H}$. This corresponds to a fair allocation of the resource between the houses. 
- Scheme 2: Each house has a different priority level. Without loss of generality, we assume that the priority coefficients satisfy: $h<h^{\prime} \Rightarrow \alpha_{h} \geq \alpha_{h^{\prime}}, \forall h, h^{\prime}=$ $1, \ldots, H$. A house with a larger priority coefficient will be served before a house with a smaller priority coefficient. In case of a tie, the house with the smaller index has priority. As a result the following priority allocation scheme holds: (priority of house 1$)>($ priority of house 2$)>\ldots>($ priority of house $H)$.

\subsection{Dynamic evolution of house $h$ temperature}

We denote as $\theta_{e}(t)$ and $\theta_{h}(t)$, respectively, the exterior temperature and the temperature of house $h$, at time period $t$. We assume that they both belong to the interval $[0 ; \Theta]$ where $\Theta<+\infty$ is a finite upper bound for the temperature fixed a priori. It can be associated with the maximum temperature of the heating system. For the sake of simplicity we let: $\Delta \theta_{h}(t)=\theta_{h}(t)-\theta_{e}(t)$ be the gradient of temperature between house $h$ temperature and the exterior temperature at time period $t$. We suppose that it remains non-negative at any time period $t$ in $[0 ; T]$. Since we do not consider separately each room in the house, the temperature corresponds to the averaged temperature over each room of the house and its evolution takes into account thermal losses. These thermal losses depend on two facts: the house insulation of its walls and roof, and the efficiency of the heating system which distributes heat throughout the house [7]. Therefore, we introduce coefficients $\left.\left.\kappa_{h} \in\right] 0 ; 1\right]$ and $\left.\left.\bar{\kappa}_{h} \in\right] 0 ; 1\right]$ which represent, respectively, the thermal loss and the heating efficiency of house $h$. House $h$ energy demand $x_{h}(t)$ can then be interpreted as the proportion of rooms that the owner decides to heat. The consideration of the extreme cases regarding the house heating system, enables us to associate physical interpretations to the values of parameters $\kappa_{h}, \bar{\kappa}_{h}$ :

- If house $h$ is not heated then $x_{h}(t)=0$. This implies that $\theta_{h}(t)=\theta_{h}(t-1)+$ $\left(\theta_{e}(t-1)-\theta_{h}(t-1)\right) \kappa_{h}$. In this case, we notice that $\theta_{h}(t)=\theta_{h}(t-1)$ if, and only if, $\kappa_{h}=0 . \theta_{h}(t)=\theta_{e}(t-1)$ if, and only if, $\kappa_{h}=1$. As a result, the more $\kappa_{h}$ approaches 1 (resp. 0), the poorer (resp. the better) is its wall and roof insulation.

- If house $h$ is heated at its maximum then $x_{h}(t)=1$. This implies that $\theta_{h}(t)=$ $\theta_{h}(t-1)+\left(\Theta-\theta_{h}(t-1)\right) \bar{\kappa}_{h}$. In this case, we notice that $\theta_{h}(t)=\theta_{h}(t-1)$ if, and only if, $\bar{\kappa}_{h}=0 . \theta_{h}(t)=\Theta$ if, and only if, $\bar{\kappa}_{h}=1$. As a result, the more $\bar{\kappa}_{h}$ approaches 1 (resp. 0), the smaller (resp. the higher) are the losses from the heating system.

Merging the extreme case outputs, we determine house $h$ temperature dynamic evolution by the recursive equation detailed below:

$$
\begin{aligned}
\theta_{h}(t) & =\theta_{h}(t-1)+\left(\theta_{e}(t-1)-\theta_{h}(t-1)\right)\left(1-x_{h}(t)\right) \kappa_{h}+(\Theta \\
& \left.-\theta_{h}(t-1)\right) x_{h}(t) \bar{\kappa}_{h}
\end{aligned}
$$


We set: $\theta_{h}(0)=\theta, \theta \in[0 ; \Theta]$. We rewrite Equation 22, factorizing it by $x_{h}(t)$ :

$\theta_{h}(t)=\underbrace{\left(\theta_{h}(t-1)-\Delta \theta_{h}(t-1) \kappa_{h}\right)}_{\geq 0}+\left(\Delta \theta_{h}(t-1) \kappa_{h}+\left(\Theta-\theta_{h}(t-1)\right) \bar{\kappa}_{h}\right) x_{h}(t)$

Lemma 1. If $\frac{\bar{\kappa}_{h}}{\kappa_{h}}>1$, the temperature in house $h$ increases linearly in the energy demand, $x_{h}(t)$.

Proof of Lemma1. According to Equation (3), the temperature in house $h$ increases linearly according to the energy demand $x_{h}(t)$ if, and only if, the leading coefficient is positive. Formally, we should have: $\Delta \theta_{h}(t-1) \kappa_{h}+\left(\Theta-\theta_{h}(t-1)\right) \bar{\kappa}_{h}>0 \Leftrightarrow$ $-\frac{\Delta \theta_{h}(t-1)}{\Theta-\theta_{h}(t-1)}<\frac{\bar{\kappa}_{h}}{\kappa_{h}}$. Two cases should be considered to determine under which assumption the above inequality holds. First, if $\Delta \theta_{h}(t-1) \geq 0$ then since $\frac{\bar{\kappa}_{h}}{\kappa_{h}}>0$, the inequality is always true. Second, if $\Delta \theta_{h}(t-1)<0$ then it is sufficient to assume that $\frac{\bar{\kappa}_{h}}{\kappa_{h}}>1$ to guarantee that the inequality holds.

To guarantee that Lemma 1 holds, we will suppose that $\frac{\bar{\kappa}_{h}}{\kappa_{h}}>1$ throughout the article.

Judging by the results derived from Equation (2) physical interpretations and by Lemma 1. we have pictured in colors the area of definition for parameters $\kappa_{h}, \bar{\kappa}_{h}$ of house $h$, in Figure 3 Area 1 corresponds to a bad heating efficiency and a good insulation of the house. In Area 2, the house's insulation is bad but its heating efficiency is high. Finally, in Area 3, both the insulation and the heating efficiency are good.

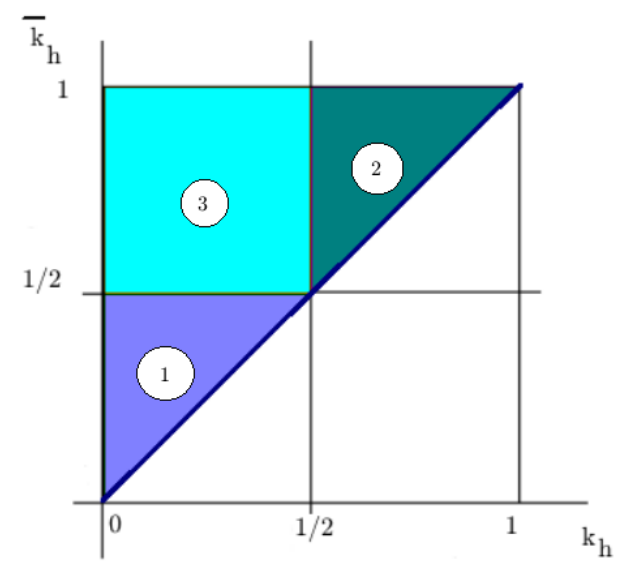

Figure 3: Areas of definition for house $h$ parameters $\kappa_{h}, \bar{\kappa}_{h}$.

In Figure 4, we have represented the quantity of energy $x_{h}($.$) , necessary to reach$ the optimal temperature in the house when the exterior temperature is at its minimum 
i.e., $\theta_{h}()=.\theta_{h}^{0}=21^{0} C$ and $\theta_{e}()=.0^{0} C$, as a function of thermal loss and heating efficiency parameters. As expected, a house with small thermal losses and high heating efficiency will require little heat to reach the optimal temperature. But this quantity increases as thermal loss increases and to lesser extent, as heating efficiency decreases. Furthermore, the impact of thermal loss is higher than the impact of heating efficiency on the definition of the quantity of energy necessary to reach the optimal temperature.

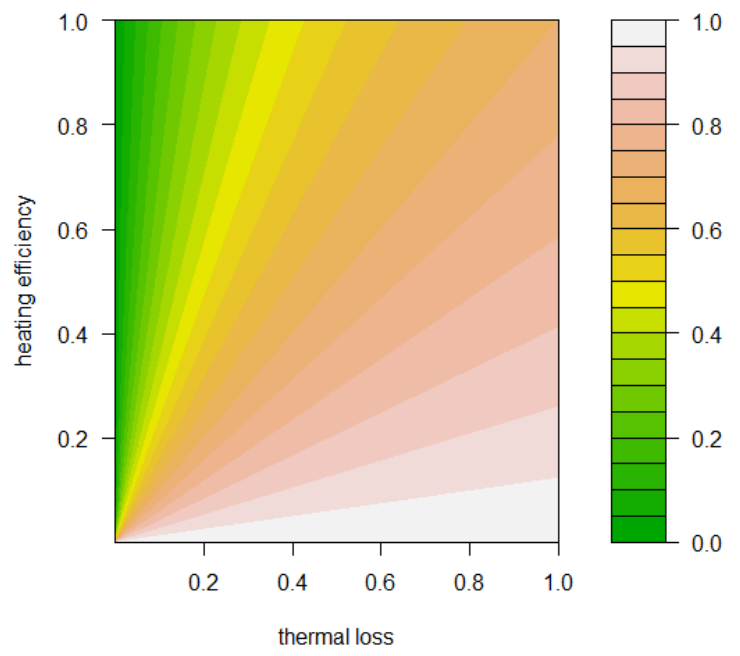

Figure 4: Quantity of energy necessary to reach the house's optimal temperature expressed as a function of thermal loss and heating efficiency when the exterior temperature reaches its minimum.

\section{No storage}

In this section, we assume that: $\Delta q_{h}(t)=0, \forall h=1, \ldots, H, \forall t \in[0 ; T]$. We describe the game steps at time period $t$. It takes the form of a Stackelberg game where the eco-neighborhood manager $E$ is the leader and the $H$ houses are the followers.

(1) Eco-neighborhood manager $E$ allocates his resource $R(t)$ between the $H$ houses so as to maximize his utility $\pi_{E}(t)$.

(2) Each house $h$ determines independently and simultaneously its energy demand $x_{h}(t)$ so as to maximize its utility $\pi_{h}(t), \forall h=1, \ldots, H$.

The game is then repeated over a finite horizon $[0 ; T]$ such that $T<+\infty$. The game can be solved analytically proceeding by backward induction. This approach is quite classical in Stackelberg settings [13]. 
Proposition 2. At any time period $t$ and for any house $h=1, \ldots, H$, there exists a unique equilibrium in $\left(x_{h}^{*}(t), R_{h}^{*}(t)\right)$ for the Stackelberg game. Furthermore, the equilibrium is efficient under priority allocation scheme.

Proof of Proposition 2. We start by Step (2) of the Stackelberg game described above i.e., the optimization of the energy demand of house $h$. We consider that the resource allocation rule is fixed. Two cases should be distinguished depending on the sign of $x_{h}(t)-R_{h}(t)$.

Case (i): $x_{h}(t) \leq R_{h}(t)$

Under this assumption, the total energy demand of house $h$ is entirely satisfied by drawing in the resource allocated by the eco-neighborhood manager $E$. In this case $\left(x_{h}(t)-R_{h}(t)\right)_{+}=0$ since $x_{h}(t)-R_{h}(t) \leq 0$. House $h$ utility simplifies to: $\pi_{h}(t)=\lambda_{h} \mathcal{C}_{h}\left(\theta_{h}(t)\right)$. Differentiating $\pi_{h}(t)$ with respect to $x_{h}(t)$, we obtain: $\frac{\partial \pi_{h}(t)}{\partial x_{h}(t)}=\underbrace{\frac{\lambda_{h}}{\sqrt{2 \pi}} \exp \left(-\frac{\left(\theta_{h}(t)-\theta_{h}^{0}\right)^{2}}{2}\right)}_{>0}\left(\theta_{h}^{0}-\theta_{h}(t)\right) \frac{\partial \theta_{h}(t)}{\partial x_{h}(t)}$.

Considering the right part of the equation, we have: $\frac{\partial \theta_{h}(t)}{\partial x_{h}(t)}=\left(\theta_{h}(t-1)-\theta_{e}(t-\right.$ 1)) $\kappa_{h}+\left(\Theta-\theta_{h}(t-1)\right) \bar{\kappa}_{h}>0$. Then, according to Lemma 1;

$$
\begin{aligned}
& \theta_{h}(t-1)-\theta_{e}(t-1)>\frac{\bar{\kappa}_{h}}{\kappa_{h}}\left(\theta_{h}(t-1)-\Theta\right) \\
\Leftrightarrow & \kappa_{h}\left(\theta_{h}(t-1)-\theta_{e}(t-1)\right)>\bar{\kappa}_{h}\left(\theta_{h}(t-1)-\Theta\right)
\end{aligned}
$$

This implies that: $\frac{\partial \theta_{h}(t)}{\partial x_{h}(t)}=\kappa_{h}\left(\theta_{h}(t-1)-\theta_{e}(t-1)\right)+\left(\Theta-\theta_{h}(t-1)\right) \bar{\kappa}_{h}>$ $\underbrace{\bar{\kappa}_{h}\left(\theta_{h}(t-1)-\Theta\right)+\left(\Theta-\theta_{h}(t-1)\right) \bar{\kappa}_{h}}_{=0}$.

Therefore:

$$
\begin{aligned}
\frac{\partial \pi_{h}(t)}{\partial x_{h}(t)}=0 & \Leftrightarrow \theta_{h}(t)=\theta_{h}^{0} \\
& \Leftrightarrow \quad x_{h}(t)=\frac{\theta_{h}^{0}-\theta_{h}(t-1)+\Delta \theta_{h}(t-1) \kappa_{h}}{\Delta \theta_{h}(t-1) \kappa_{h}+\left(\Theta-\theta_{h}(t-1)\right) \bar{\kappa}_{h}}
\end{aligned}
$$

Case (ii): $x_{h}(t)>R_{h}(t)$

Under this assumption, the eco-neighborhood manager $E$ cannot satisfy the entire demand of house $h$. This latter will need to buy the lacking energy to the service provider who provides energy to the microgrid. House $h$ utility then takes the form: $\pi_{h}(t)=\lambda_{h} \mathcal{C}_{h}\left(\theta_{h}(t)\right)-p(t)\left(x_{h}(t)-R_{h}(t)\right)$. Differentiating $\pi_{h}(t)$ with respect to 
$x_{h}(t)$, the equation is equivalent to the derivative equals 0 :

$$
\left(\theta_{h}^{0}-\theta_{h}(t)\right) \underbrace{\frac{\partial \theta_{h}(t)}{\partial x_{h}(t)}}_{>0}=\frac{p(t) \sqrt{2 \pi}}{\lambda_{h}} \exp \left(\frac{\left(\theta_{h}(t)-\theta_{h}^{0}\right)^{2}}{2}\right)
$$

According to Case (i) studied above, $\frac{\partial \theta_{h}(t)}{\partial x_{h}(t)}>0$. The left part of Equation 4 is linearly decreasing in $\theta_{h}(t)$ and vanishes in $\theta_{h}(t)=\theta_{h}^{0}$. Computing the differentiate of the right part of Equation (4) with respect to $\theta_{h}(t)$, we obtain that it is decreasing until $\theta_{h}(t)=\theta_{h}^{0}$ and increasing for $\theta_{h}(t)>\theta_{h}^{0}$. Furthermore, the right part is positive in $\theta_{h}^{0}$ where it equals $\frac{p(t) \sqrt{2 \pi}}{\lambda_{h}}$. Judging by both function shapes, we infer that the game admits a unique equilibrium in $x_{h}^{*}(t)$ if, and only if:

$$
\left.\theta_{h}^{0} \frac{\partial \theta_{h}(t)}{\partial x_{h}(t)}\right|_{\theta_{h}(t)=0} \geq \frac{p(t) \sqrt{2 \pi}}{\lambda_{h}} \exp \left(\frac{\theta_{h}^{02}}{2}\right)
$$

Now, we will assume that there exists reals $\left.\left.r, r^{\prime} \in\right] 0 ; 1\right]$ such that $\theta_{h}^{0}=r \Theta$ and that the maximum exterior temperature can be written as: $r^{\prime} \Theta$. These points can be justified by the fact that the optimal temperature in house $h$ is necessarily smaller than the maximum temperature that can be reached by the heating system and by the fact that the maximum temperature of the heating system should be larger than the maximum exterior temperature since otherwise our model would be inapplicable to countries where the temperature remains low. Additionally, we make the assumption that the unit energy price $p(t)$, varies in interval $\left[0 ; p_{M}\right]$ where $p_{M}$ is the price upper bound. This is as if the State or an unbiased regulator, fixed a price cap to avoid situations of abuse of monopoly or tacit collusion between the service providers which might generate an artificially high energy price. Such an assumption is quite classical in industrial organization economics. Then, choosing the game parameters so that:

$$
r^{\prime} \leq 1-\frac{p_{M} \sqrt{2 \pi}}{\lambda_{h} r \Theta^{2} \kappa_{h}} \exp \left(\left(\frac{1}{\sqrt{2}} r \Theta\right)^{2}\right)
$$

guarantees that Inequality (5) holds and a fortiori, that the game admits a unique equilibrium in $x_{h}^{*}(t)$.

The energy demand being optimized, we skip to Step (1) of the Stackelberg game i.e., the optimization of the resource allocation. As explained at the end of Section 2 , we consider two schemes defining the allocation rule: either the allocation is fair or a priority allocation scheme holds.

Under a fair scheme, the resource available in the eco-neighborhood is allocated so that: $R_{h}^{*}(t)=\frac{R(t)}{H}, \forall h=1, \ldots, H$.

Under a priority allocation scheme, the rule becomes:

- For house 1

$$
R_{1}^{*}(t)=\left\{\begin{aligned}
x_{1}^{*}(t) & \text { if } x_{1}^{*}(t) \leq R(t) \\
R(t) & \text { otherwise }
\end{aligned}\right.
$$


- For house $h=2, \ldots, H$

$$
R_{h}^{*}(t)=\left\{\begin{aligned}
x_{h}^{*}(t) & \text { if } x_{h}^{*}(t) \leq R(t)-\sum_{l=1}^{h-1} R_{l}^{*}(t), \\
R(t)-\sum_{l=1}^{h-1} R_{l}^{*}(t) & \text { otherwise. }
\end{aligned}\right.
$$

By definition, the Pareto frontier of the game is the set of points such that once one of them is reached, no agent's utility can be increased without decreasing at least one agent's utility [13]. In the game without storage, the Pareto frontier containing the set of efficient equilibria is defined by the following equations: $R_{h}(t) \leq x_{h}(t), \forall h=$ $1, \ldots, H$ and $\sum_{h=1, \ldots, H} R_{h}(t)=R(t)$. As a result, the equilibrium obtained under a priority allocation scheme is efficient whereas it is not in general under a fair allocation scheme. Of course the priority allocation scheme is not the unique resource allocation scheme that guarantees the reaching of a Pareto optimum.

\section{Storage under stochastic environment}

Most of the energy produced autonomously by the eco-neighborhood comes from solar panels deployed on the roofs and on the fronts of the eco-neigborhood houses. Empirical observations on real data and physical relations which describe the dependence on temperature of photovoltaic module performance [20] lead us to assume that there exists a bijective relation between the energy produced by the eco-neighborhood and the exterior temperature i.e., there exists a bijective function $\varphi($.$) from [0 ; \Theta]$ to $[0 ; 1]$ such that:

$$
R(t)=\varphi\left(\theta_{e}(t)\right)
$$

\subsection{Resource uncertainty model}

In the literature, many articles model the weather evolution as a stationary finite discrete Markov chain [4] or use Hidden Markov Models in case where states cannot be directly observed but information about the underlying hidden state sequence is available [6]. The direct relationship between weather and energy production justifies the introduction of a stationary finite discrete Markov chain to model the uncertainty associated with the production of energy in the eco-neighborhood. Depending on outside factors such as weather conditions, we allow to have $K \in \mathbb{N}^{*}$ realizations of the produced energy. We define $p_{i j}$ as the probability that the energy produced by the eco-neighborhood will be in state $j$ in the next time period if currently it is in state $i$. 


\subsection{Problem formulation}

In this subsection, we detail the stochastic optimization problems that the houses have to face with, under a fair allocation of the resource in Subsection 4.2.1 and with a priority mechanism in Subsection 4.2.2

\subsubsection{Allocation scheme 1: The $H$ houses have equal priority}

We define a generic state of the system as: $s_{H}=\left(R, \theta_{1}, Q_{1}, \theta_{2}, Q_{2}, \ldots, \theta_{H}, Q_{H}\right) \in$ $\{1,2, \ldots, K\} \times\left([0 ; \Theta] \times\left[0 ; Q_{\max }\right]\right)^{H}$. We let $S=\{1,2, \ldots, K\} \times\left([0 ; \Theta] \times\left[0 ; Q_{\max }\right]\right)^{H}$ be the set of all possible states $s_{H}$. At any time period $t$ the system being in state $s_{H}$, we want to choose an action for each house $h=1, \ldots, H$ from the set of allowable actions in state $s_{H}, A_{s_{H}}$. Actions are the energy demand and the variation of the storage for each of the $H$ houses in each stage with different realizations of the resource produced by the eco-neighborhood. We denote as: $a_{h}(t)=\left(x_{h}(t), \Delta q_{h}(t)\right)$ the action chosen by house $h$ at time period $t$. When in state $s_{H}$, the set of admissible actions obtained after reordering Equation (2) and considering the storage constraints defined in Equation (1) is denoted: $A_{s_{H}}$.

In our optimization problem, each house $h$ wants simultaneously and independently to maximize the expected discounted sum of its utilities over time interval $[0 ; T]$. We introduce $\delta \in] 0 ; 1]$ as the discount factor of our stochastic optimization problem [15]. The more $\delta$ approaches 1 , the more house $h$ is sensitive to the future or has a long-term vision. Reciprocally, the more $\delta$ approaches 0 , the shorter is the vision of house $h$. For any house $h=1, \ldots, H$, the optimization problem can be written as:

$$
\max _{a_{h} \in A_{s_{H}}} \mathbb{E}\left[\left.\sum_{t=0}^{T} \delta^{t} \pi_{h}(t)\right|_{s_{H}, a_{h}}\right]
$$

under an equal allocation of the resource i.e.: $R_{h}^{*}(t)=\frac{R(t)}{H}, \forall t=0,1, \ldots, T$.

\subsubsection{Allocation scheme 2 : (priority of house 1$)>($ priority of house 2$)>\ldots>($ priority of house $H$ )}

This is a sequential optimization problem. The steps are the following.

Step 1: In this step, we assume that there is only a single house in the econeighborhood and that is house 1 , which has the highest priority level. The state of the optimization problem is defined as $s_{1}=\left(R, \theta_{1}, Q_{1}\right) \in S_{1}=\{1,2, \ldots, K\} \times$ $[0 ; \Theta] \times\left[0 ; Q_{\max }\right]$, where $R$ contains the energy produced by the eco-neighborhood, $\theta_{1}$ is the temperature and $Q_{1}$ the storage level in house 1 . If we decide to choose an action $a_{1}=\left(x_{1}, \Delta q_{1}\right) \in A_{s_{1}}$ at time period $t$, house 1 receives $\left.\pi_{1}(t)\right|_{s_{1}, a_{1}}$ as utility. We optimize the following problem:

$$
\max _{a_{1} \in A_{s_{1}}} \mathbb{E}\left[\left.\sum_{t=0}^{T} \delta^{t} \pi_{1}(t)\right|_{s_{1}, a_{1}}\right]
$$


The allocation rule at time period $t$ is:

$$
R_{1}^{*}(t)=\left\{\begin{aligned}
x_{1}^{*}(t)+\Delta q_{1}^{*}(t) & \text { if } x_{1}^{*}(t)+\Delta q_{1}^{*}(t) \leq R(t), \\
R(t) & \text { otherwise. }
\end{aligned}\right.
$$

Step 2: In this step, we consider house 2 and house 1. House 2 has the second highest priority level. The state of the optimization problem is $s_{2}=\left(R, \theta_{1}, Q_{1}, \theta_{2}, Q_{2}\right) \in$ $S_{2}=\{1,2, \ldots, K\} \times\left([0 ; \Theta] \times\left[0 ; Q_{\max }\right]\right)^{2}$, where $\theta_{2}$ is the temperature and $Q_{2}$ the storage level in house 2 . At any stage $t$, we choose an action $a_{2}=\left(x_{2}, \Delta q_{2}\right) \in A_{s_{2}}$ so as to optimize the following problem:

$$
\max _{a_{2} \in A_{s_{2}}} \mathbb{E}\left[\left.\sum_{t=0}^{T} \delta^{t} \pi_{2}(t)\right|_{s_{2}, a_{2}}\right]
$$

We describe the allocation rule at time period $t$ under priority scheme 2 , for both houses. For house 1, we have:

$$
R_{1}^{*}(t)=\left\{\begin{aligned}
x_{1}^{*}(t)+\Delta q_{1}^{*}(t) & \text { if } x_{1}^{*}(t)+\Delta q_{1}^{*}(t) \leq R(t), \\
R(t) & \text { otherwise. }
\end{aligned}\right.
$$

And, for house 2, we have:

$$
R_{2}^{*}(t)=\left\{\begin{array}{cl}
x_{2}^{*}(t)+\Delta q_{2}^{*}(t) & \text { if } x_{2}^{*}(t)+\Delta q_{2}^{*}(t) \leq R(t)-R_{1}^{*}(t), \\
R(t)-R_{1}^{*}(t) & \text { otherwise }
\end{array}\right.
$$

Steps 3 to $H$ : We keep following the same procedure until we have solved for the $H$ houses. The state of the optimization problem is $s_{H}=\left(R, \theta_{1}, Q_{1}, \ldots, \theta_{H}, Q_{H}\right) \in$ $S=\{1,2, \ldots, K\} \times\left([0 ; \Theta] \times\left[0 ; Q_{\max }\right]\right)^{H}$, where $\theta_{h}$ is the temperature and $Q_{h}$ the storage level in any house $h=1, \ldots, H$. At any stage $t$, the $H$-th house which has the lowest priority, chooses an action $a_{H}=\left(x_{H}, \Delta q_{H}\right) \in A_{s_{H}}$ so as to optimize the following problem:

$$
\max _{a_{H} \in A_{s_{H}}} \mathbb{E}\left[\left.\sum_{t=0}^{T} \delta^{t} \pi_{H}(t)\right|_{s_{H}, a_{H}}\right]
$$

We describe the allocation rule at time period $t$ under priority scheme 2 , for the $H$ houses. For house 1, we have:

$$
R_{1}^{*}(t)=\left\{\begin{aligned}
x_{1}^{*}(t)+\Delta q_{1}^{*}(t) & \text { if } x_{1}^{*}(t)+\Delta q_{1}^{*}(t) \leq R(t), \\
R(t) & \text { otherwise. }
\end{aligned}\right.
$$

And, for any house $h=2, \ldots, H$, we have:

$$
R_{h}^{*}(t)= \begin{cases}x_{h}^{*}(t)+\Delta q_{h}^{*}(t) & \text { if } x_{h}^{*}(t)+\Delta q_{h}^{*}(t) \leq R(t)-\sum_{l=1}^{h-1} R_{l}^{*}(t), \\ R(t)-\sum_{l=1}^{h-1} R_{l}^{*}(t) & \text { otherwise. }\end{cases}
$$




\subsection{Markov decision processes}

There exist multiple ways to find or at least, to approximate, the optimal strategy i.e., the optimal sequence of actions, which maximizes the agents' utilities. A first possibility is to learn it, by making the agents interact in their environment and adapt dynamically their actions while learning the strategy of the other agents. Many algorithms have been developed in this direction using adaptive dynamic programming [14], Q-learning, and more generally reinforcement learning [21], [24] and artifical intelligence techniques. A second class of methods consists in planning off-line the optimal sequence of actions to play for each possible initial state [3], [15]. The (learnt or planned) optimal strategies will coincide with the equilibria of the stochastic game.

In this article, we choose to focus on the second category of methods and to solve the sequential stochastic optimization problem introduced in Subsection 4.2. We associate a $T$-horizon Markov Decision Process (MDP) for each house $h$. The dynamic evolution of this process is governed by a control strategy which is optimized simultaneously and independently by each house. House $h T$-horizon control strategy will be denoted: $\bar{a}_{h}=\left(\bar{a}_{h}(0), \bar{a}_{h}(1), \ldots, \bar{a}_{h}(T)\right)$. At any time period $t=0,1, \ldots, T$, $\bar{a}_{h}(t)=\left(\bar{a}_{h}\left(t, s_{H}\right)\right)_{s_{H} \in S}$ is a vector containing the optimal action to choose in state $s_{H} \in S . \bar{a}_{h}(t)$ belongs to the set of stationary strategies and $\bar{a}_{h}$ to the set of Markov strategies of the $T$-horizon MDP: $\mathcal{F}_{M}^{T}$.

The $T$-stage value of the control strategy $\bar{a}_{h}$ is: $\mathbb{E}_{\bar{a}_{h}}\left[\left.\sum_{t=0}^{T} \delta^{t} \pi_{h}(t)\right|_{s_{H}(0)=s_{H}}\right]$ for every initial state $s_{H} \in S$, where $\mathbb{E}_{\bar{a}_{h}}[$.$] is the expectation taken with respect to house$ $h$ control strategy: $\bar{a}_{h}$. The optimization problem for house $h$ becomes:

$$
\max _{\bar{a}_{h} \in \mathcal{F}_{M}^{T}}\left(\mathbb{E}_{\bar{a}_{h}}\left[\left.\sum_{t=0}^{T} \delta^{t} \pi_{h}(t)\right|_{s_{H}(0)=s_{H}}\right]\right)_{s_{H} \in S}
$$

House $h$ transition probabilities Let us consider two states: $s_{H}=\left(i, \theta_{1}, Q_{1}, \ldots\right.$, $\left.\theta_{H}, Q_{H}\right) \in S$ and $s_{H}^{\prime}=\left(i^{\prime}, \theta_{1}^{\prime}, Q_{1}^{\prime}, \ldots, \theta_{H}^{\prime}, Q_{H}^{\prime}\right) \in S$. Equation (2) enables us to define two applications: $\psi:\left(i, \theta_{h}, a_{h}\right) \mapsto \theta_{h}^{\prime}$ and $\tilde{\psi}:\left(i, \theta_{h}, \theta_{h}^{\prime}\right) \mapsto x_{h}$ which define in a formal way the temperature evolution and the restriction on the action to be chosen depending on the state transition, respectively. Additionally, each house $h$ has beliefs on the actions of the other houses in the eco-neighborhood. These beliefs are defined a priori in the game. Indeed, the choice of action being simultaneous, house $h$ does not observe the other houses' actions. For any house $l=1, \ldots, H, l \neq h$ in the eco-neighborhood, house $h$ belief regarding the action that house $l$ might choose is represented by a $|A|$-dimensional vector: $\rho_{h l}$ which is a probability distribution defined over the action set $A=\cup_{s_{H} \in S} A_{s_{H}}$. Using the independence assumption on the houses' choices and Bayes formula, we derive house $h$ transition probability from state 
$s_{H}$ to state $s_{H}^{\prime}$ conditionally on action $a_{h}$ :

$$
\left.p^{h}\left(s_{H}^{\prime} \mid s_{H}, a_{h}\right)=1_{\left\{\psi\left(i, \theta_{h}, a_{h}\right)=\theta_{h}^{\prime}\right.}\right\}^{p_{i i^{\prime}}} \prod_{l=1, \ldots, H, l \neq h} \sum_{a_{l} \in\left\{A \mid x_{l}=\tilde{\psi}\left(i, \theta_{l}, \theta_{l}^{\prime}\right)\right\}} \rho_{h l}\left(a_{l}\right)
$$

Then for any house $h=1, \ldots, H$, the transition probabilities conditionally on action $a_{h} \in A$ are stored in a matrix: $P^{h, a_{h}}=\left(p^{h}\left(s_{H}^{\prime} \mid s_{H}, a_{h}\right)\right)_{s_{H}, s_{H}^{\prime} \in S}$.

The algorithm The generic notation $s$ is used to refer to a state because depending on the considered allocation scheme, it can be $2,3, \ldots, H$, or $H+1$-dimensional. The principle of optimality leads us to solve the problem using backward recursion of dynamic programming [3], [15]:

For each $t=1,2, \ldots, T$, we calculate for each state $s \in S$ the optimal action to choose as the solution of the optimization problem:

$$
\bar{a}_{h}^{*}(T-t, s)=\arg \max _{a_{h} \in A}\left\{\left.\pi_{h}(.)\right|_{s, a_{h}}+\sum_{s^{\prime} \in S} p^{h}\left(s^{\prime} \mid s, a_{h}\right) V_{t-1}^{h}\left(s^{\prime}\right)\right\}
$$

For any house $h=1, \ldots, H$, we let: $V_{t}^{h}(s), \forall s \in S$ be the value of the game for house $h$ in any state $s$ at time period $t$. It is updated at each time period $t$ :

$$
V_{t}^{h}(s)=\left.\pi_{h}(.)\right|_{s, \bar{a}_{h}^{*}(T-t, s)}+\sum_{s^{\prime} \in S} p^{h}\left(s^{\prime} \mid s, \bar{a}_{h}^{*}(T-t, s)\right) V_{t-1}^{h}\left(s^{\prime}\right)
$$

The algorithm of backward recursion of dynamic programming for house $h=1, \ldots, H$ will be called Algorithm BR. We detail below the algorithm that will be used in Section 5 to simulate the system evolution under the allocation scheme 2 , described in Subsection 4.2.2.

Simulation of the eco-neighborhood energy consumption under scheme?

(1) Recursion.

- House 1 . For any $a_{1}$, house 1 computes the conditional probability transition matrix $P^{1, a_{1}}$ taking into account only house 1 . Algorithm BR is run and gives the optimal action to choose in each state $s_{1}=\left(R, \theta_{1}, Q_{1}\right)$ at any time period. As the output we obtain the optimal control strategy for house 1: $\bar{a}_{1}^{*}$.

- House 2 To $H$. For any $a_{h}$, house $h=2, \ldots, H$ computes the conditional probability transition matrix $P^{h, a_{h}}$ taking into account only houses $1, \ldots, h$. Algorithm BR is run and gives the optimal action to choose in each state $s_{h}=\left(R, \theta_{1}, Q_{1}, \ldots, \theta_{h}, Q_{h}\right)$ at any time period. As the output we obtain the optimal control strategy for house $h: \bar{a}_{h}^{*}$.

(2) Simulation of the eco-neighborhood energy consumption.

- Time Period 1 To $T$. Given the system initial state $s_{H}(0) \in S$, from time period 1 to $T-1$, the system is in state $s_{H}(t) \in S$. The optimal action to choose for each house $h=$ $1, \ldots, H$ is contained in $\bar{a}_{h}^{*}\left(t, s_{H}(t)\right)$. The energy produced by the eco-neighborhood evolves according to the Markov chain having transition matrix $\left(p_{i, j}\right)_{i, j=1, \ldots, K}$, generating $R(t+1)$. It is allocated between the houses according to the priority rule described in 4.2 .2 
- The system ends in state $s_{H}(T) \in S$.

On the contrary, under the allocation scheme 1 described in Subsection 4.2.1, the resource allocation mechanism is known at the beginning of each time period, by every house. As a result, under the allocation scheme 1, the algorithm begins with an inititialization step where each house $h=1, \ldots, H$ computes its conditional probability transition matrix taking into account all the other houses. Then, in the recursion step, each house runs simultaneously and independently Algorithm BR since there is no priority rule. The simulation of the system evolution remains unchanged except that each house $h=1, \ldots, H$ receives an equal share: $\frac{R(t)}{H}$ of the resource.

\section{Economic guidelines for the operations of the eco- neighborhood}

The aim of this section is to determine whether houses in the eco-neighborhood, have economic incentives to store energy under various pricing mechanisms and heterogeneity in their characteristics. To perform this study, we start by simulating the econeighborhood consumption over a finite time horizon using the algorithm introduced in Subsection 4.3 and then, we plot the resulting cumulative discounted utilities of the houses assuming that they have either storage capacity or no storage capacity. The derived results will enable us to determine whether storage is a natural issue for the eco-neighborhood, which means that it should be worth for politics investing in the development of even more efficient storage systems or, on the contrary, elaborating more sophisticated economic incentives in the form of contracts or pricing mechanisms, to promote it.

\subsection{Comparisons of the agents' incentives to store energy under various pricing mechanisms}

In this subsection, we consider two houses in the eco-neighborhood i.e., $H=2$ and $K=11$ outcomes for the resource. Both the energy demand and the storage belong to the interval $[0 ; 1]$ discretized according to a 0.2 step-size. We choose $\Theta=40^{\circ} \mathrm{C}$ and $3^{\circ} \mathrm{C}$ as discretization step for the temperature interval. The resource generating transition matrix is defined so that: $p_{i i}=\frac{0.8}{11}+0.2$ and $p_{i i^{\prime}}=\frac{0.8}{11}, \forall i^{\prime} \neq i$. The comfort sensitivity parameters are chosen so that: $\lambda_{1}=150, \lambda_{2}=200$ i.e., house 2 is more sensitive to its comfort than house 1 . The optimal temperature for each house is set to the most common values: $\theta_{1}^{0}=21^{\circ} \mathrm{C}$ and $\theta_{2}^{0}=19^{\circ} \mathrm{C}$. The discount factor is fixed at: $\delta=0.7$. Beliefs are uniform for house 2. This assumption is required when the planning algorithm described in Subsection 4.3 is run and house 2 needs to compute its conditional probability transition matrix. In this subsection, the houses' thermal loss and heating efficiency parameters are supposed homogeneous within the houses i.e., $\kappa_{h}=0.3, \bar{\kappa}_{h}=0.7, \forall h=1,2$. In this subsection, we compare the agents' cumulative discounted utilities over a finite $T$ horizon, under the four pricing mechanisms proposed by the French electricity company [26], adapted to our study. The horizon of 
the game is fixed at: $T=100$. It is supposed to coincide with the spanning of 50 days, the houses having the opportunity to adapt their energy demand and stock level twice a day i.e., at 8 a.m. and at 11 p.m.

\subsubsection{Flat rate}

In this pricing scheme, the price is constant: $p(t)=12.49, \forall t=0,1, \ldots, T$. This value is fixed by the French electricity company as one option among its publicly available tariff schedules [26].

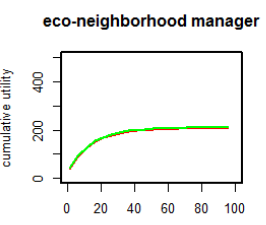

time

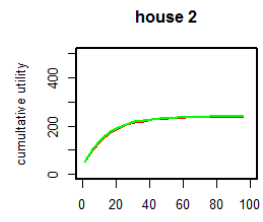

time house 1

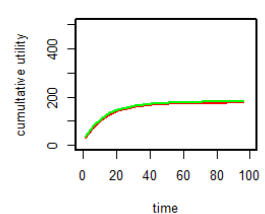

time

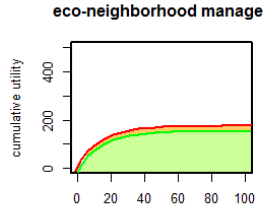

time

house 2

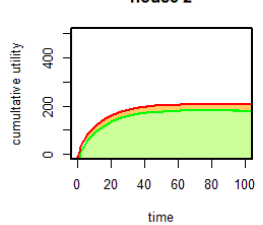

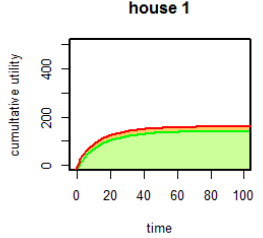

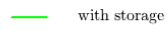

— without storage

(a)

(b)

Figure 5: Agents' cumulative discounted utilities under scheme 1 (a) and under scheme 2 (b) with flat rate pricing.

We have represented the agents' cumulative discounted utilities under an equal allocation of the resource in Figure 5 (a) and under the priority allocation scheme in Figure 5 (b) while the price is fixed according to a flat rate. In all the simulations, the cumulative discounted utilities are plotted in green when the houses have the opportunity to store energy and in red when they lack this opportunity. We observe that the agents are indifferent between both storage strategy under an equal allocation and weakly prefer not to store over to store under a priority based allocation. Therefore, under flat rate pricing, it is reasonable to state that the agents are indifferent between both storage strategies.

\subsubsection{Peak and off-peak hour pricing}

In this pricing scheme, the price can take two values depending on the hour of the day: $p(t)=13.53$ in peak hours and $p(t)=9.26$ in off-peak hours. The peak hour period runs from 6 a.m. to $10 \mathrm{p} . \mathrm{m}$. In the remaining hours, the price is fixed at the off-peak hour tarif. The principle of this pricing scheme is pictured in Figure 6 . 


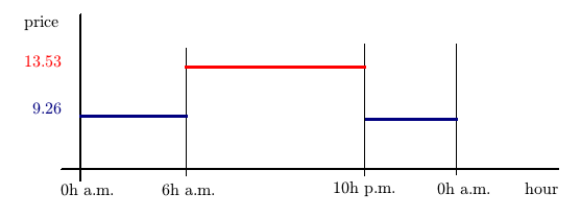

Figure 6: Peak and off-peak hour pricing principle.

We have represented the agents' cumulative discounted utilities under equal allocation in Figure 7 (a) and under a priority allocation in Figure 7(b). We observe that under both allocation schemes, the capacity to store energy enables the agents to increase their discounted cumulative utilities compared to the case without storage.

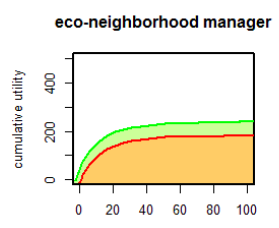

time

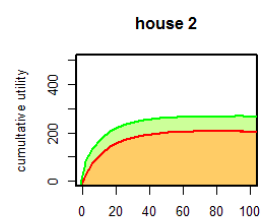

time
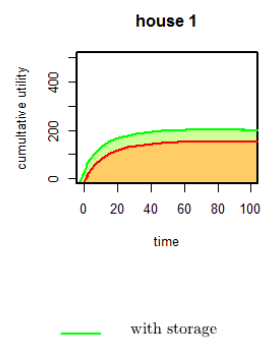

without storage

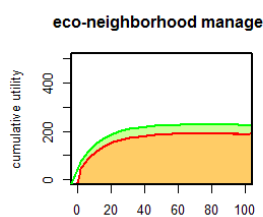

time

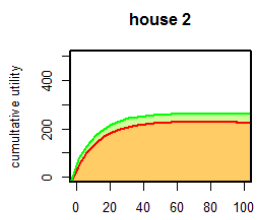

time

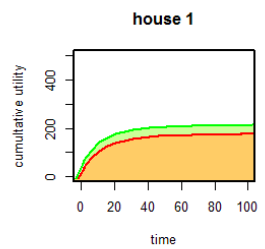

time

(a)

(b)

Figure 7: Agents' cumulative discounted utilities under scheme 1 (a) and under scheme 2 (b) with peak and off-peak hour pricing.

\subsubsection{Blue/White/Red day pricing}

In this pricing scheme, the price can take six values depending on the color of the day and of the hour of the day: in blue days, $p(t)=8.69$ in peak hours and $p(t)=7.25$ in off-peak hours ; in white days, $p(t)=12.34$ in peak hours and $p(t)=10.36$ in off-peak hours ; in red days, $p(t)=50.81$ in peak hours and $p(t)=19.33$ in off-peak hours.

Under an equal allocation of the resource, we observe in Figure 8 (a) that the agents are indifferent between both storage strategy. When priority is introduced as pictured in Figure 8 (b), we observe that the capacity to store energy is more profitable for the agents than in case where they lack it. 


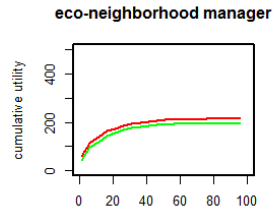

time

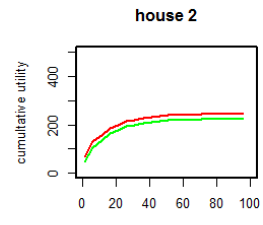

time

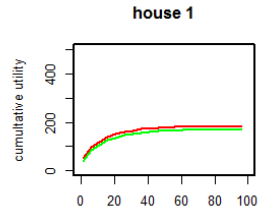

time

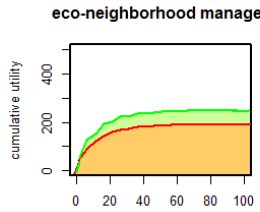

time

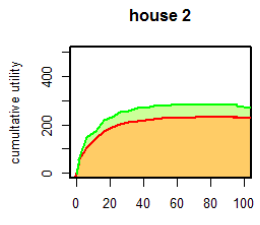

time

(a)
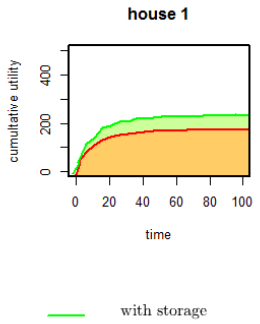

- without storage

Figure 8: Agents' cumulative discounted utilities under scheme 1 (a) and under scheme 2 (b) with Blue/White/Red day pricing.

\subsubsection{Peak day clearing pricing}

In this pricing scheme, the price can take two values depending whether the day is considered as normal or as a peak day. In the first case $p(t)=10.30$ and in the second case, $p(t)=53.29$. The peak days are mobile and allocated randomly. The only information that is available is that there are 22 peak days over the studied period of time. This means that a day has a probability of $\frac{22}{50}$ to be a peak day. To explain how we conducted our experiment, we have generated peak days according to a multinomial density function of parameters 50 and $\frac{22}{50}$.

In Figure 9 (a), we have represented the agents' cumulative discounted utilities under an equal allocation of the resource. We observe that the agents are indifferent between the storage and the no storage capacity. In Figure 9 (b), we have plotted the agents' cumulative discounted utilities under a priority allocation of the resource. We observe the the capacity to store energy is more profitable for the agents than no storage.

\subsubsection{Summary of the results}

In Table 1, we have indicated which storage policy between no storage, storage and indifference between the two, is more profitable for the agents depending on the chosen pricing mechanism and resource allocation scheme. We conclude that the pricing mechanism based on peak/off-peak hours is the most adapted mechanism for an economic implementation because under both resource allocation mechanism, houses will have incentives to store energy. Furthermore, this choice will not generate any debate about the resource allocation scheme to implement. This is all the more interesting as there are many debates about the implementability of priority based resource allocation mechanisms in the smart grid and the peak/off-peak hour pricing is the sole pricing 


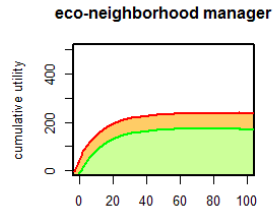

time

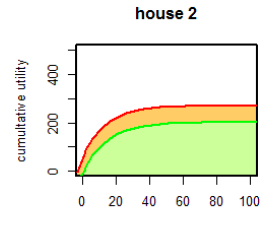

time

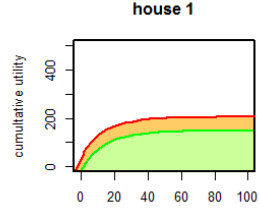

time

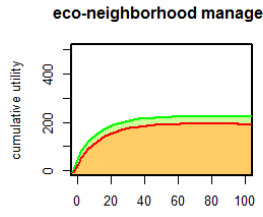

time

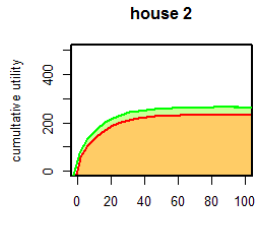

time

(a)

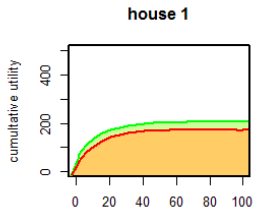

time

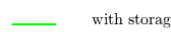

without storage

Figure 9: Agents' cumulative discounted utilities under scheme 1 (a) and under scheme 2 (b) with peak day clearing pricing.

\begin{tabular}{|c|c|c|}
\hline Allocation scheme & equal & priority \\
\hline Pricing mechanism & Indifference & Indifference \\
Flat rate & Storage & Storage \\
Peak/Off-peak & Indifference & Storage \\
Blue/White/Red & No Storage & Storage \\
\hline
\end{tabular}

Table 1: Best storage policy under different pricing mechanisms and resource allocation schemes.

mechanism which guarantees that storage is more profitable under both resource allocations. Besides, the above simulation outputs do not depend on the variablility of the comfort sensitivity parameter.

The above simulations have been realized over houses which are homogeneous in their characteristics $\left(\kappa_{h}, \bar{\kappa}_{h}, \forall h=1,2\right)$. Is the conclusion still the same for houses which are heterogeneous in their thermal loss and heating efficiency parameters?

\subsection{Impact of the heterogeneity in the houses' profiles on the in- centives to store energy}

In this subsection, we consider three profiles for the houses depending on parameters $\kappa_{h}, \bar{\kappa}_{h}$ values while the optimal temperature is supposed identical for all the houses i.e., $\theta_{h}=\theta_{h}^{0}, \forall h=1,2$. We describe the three profiles that we consider for the houses:

- Profile $A: \kappa_{h}=0.3, \bar{\kappa}_{h}=0.7$ i.e., thermal losses are small and heating efficiency is high. It belongs to area 3, as pictured in Figure 3 
- Profile $B: \kappa_{h}=0.3, \bar{\kappa}_{h}=0.4$ i.e., thermal losses are small but heating efficiency is low. It belongs to area 1, as pictured in Figure 3 .

- Profile $C: \kappa_{h}=0.6, \bar{\kappa}_{h}=0.7$ i.e., thermal losses are high and heating efficiency is high. It belongs to area 2, as pictured in Figure 3 .

We run the algorithm described in Section 4 with different priority order between the house profiles, using equal and priority based resource allocation mechanisms. The pricing mechanism is based on peak and off-peak hour pricing as described in Subsection 5.1.2. Like in Subsection 5.1, we have simulated the agents' discounted cumulative utilties as functions of time with storage capacity and without storage capacity. Depending on the discounted cumulative utility values, we determine which policy between storage, no storage and indifference between the two, is the best i.e., maximize the discounted cumulative utilities of the agents. In Table 2, we have listed the best storage policy for the agents.

\begin{tabular}{|c|c|c|}
\hline $\begin{array}{ll}\text { Priority order } & \text { Allocation scheme } \\
\end{array}$ & equal & priority \\
\hline (priority of Profile $A$ ) $>$ (prio & Indifference & Indiffe \\
\hline of Profile & Sto & No Storage \\
\hline f Profile & Sto & Indifference \\
\hline (priority of Profile $B$ ) & Storage & Indifference \\
\hline
\end{tabular}

Table 2: Best storage strategy with heterogeneity in the house profiles.

First, we observe that the influence of $\bar{\kappa}_{h}$ is predominant over the influence of $\kappa_{h}$. Second, houses have incentives to store when (priority of Profile $C$ ) $>$ (priority of Profile $A$ ) and (priority of Profile $B)>$ (priority of Profile $C$ ) and when resource allocation is equal. To avoid the case where (priority of Profile $A$ ) $>$ (priority of Profile $C$ ), it is enough to impose that the house corresponding to Profile $C$ is always served before the house corresponding to Profile $A$. To avoid the case where (priority of Profile $A$ ) > (priority of Profile $B$ ), the house having the lowest heating efficiency should invest so has to increase it.

To conclude, if any house has a poor heating system efficiency, storing energy is a waste for it since the house has to heat at maximum all the time to avoid a very bad comfort. If all houses have good heating systems, then heterogeneity in insulation can generate low incentives for energy storage if the energy allocation in the microgrid is too much inequal. Otherwise, the houses have always an incentive to store energy.

\subsection{Dynamic pricing}

In this subsection, the game parameters are identical to the ones introduced at the beginning of Subsection 5.1. The market game coincides with the one considered in [9]: 
the eco-neighborhood studied in this article is assimilated to a microgrid to which energy is supplied by a unique service provider. As already mentioned, this assumption holds well if we consider local or regional utility companies for example [9]. The energy producer can be associated with a nuclear plant, a photovoltaic park manager, a wind farm administrator, etc. His production at time period $t$ is stored in: $\nu^{e}(t)$. At time period $t$, the energy needs of the microgrid $\nu^{s}(t)$, should coincide with the sum of the consumptions of all the houses in the eco-neighborhood. The microgrid has the possibility to find alternative sources of energies for $a(t)$ energy units at a cost $\frac{a(t)^{2}}{2} 4^{4}$ Additionally, penalties which are proportional to the difference between the initially booked quantity of energy and the finally delivered quantity, are imposed from the microgrid to the service provider according to the rule: $\gamma\left(\nu^{s}(t)-a(t)-\nu^{e}(t)\right)$ + where $\gamma=0.9$ is the penalty coefficient. As proved analytically in [9], the optimal price for the service provider is: $p(t)=\frac{\nu^{s}(t)+\gamma}{2}$. The planning algorithm described in Subsection 4.3 relies on the service provider's price evolution and each house realizes a forecast $\hat{\nu}^{s}(t)$, on the price evolution by estimating the global consumption of the eco-neighborhood. We make the simplifying assumption that each house believes that the other houses will choose the same demand and storage level 55 Hence, when house $h=1, \ldots, H$ chooses action $\left(x_{h}(t), \Delta q_{h}(t)\right)$, its estimate of the microgrid's energy needs is: $\hat{\nu}^{s}(t)=H\left(x_{h}(t)+\Delta q_{h}(t)\right)-R(t)$ which leads to $\frac{H\left(x_{h}(t)+\Delta q_{h}(t)\right)-R(t)+\gamma}{2}$ as estimated price for the service provider. But, to simulate the consumption of the eco-neighborhood, the service provider's price should depend on the houses' true consumption. To compensate for the gap between the service provider's price obtained by each house independently in the planning part and in the simulation part of the algorithm described in Subsection 4.3, we introduce an error coefficient: $0 \leq \varepsilon \leq 2 H$ such that: $\nu^{s}(t)=\sum_{h=1, \ldots, H}\left(x_{h}(t)+\Delta q_{h}(t)-R_{h}(t)\right)_{+}+\varepsilon$. The higher $\varepsilon$ is, the worst are the service provider's predictions in the microgrid's energy needs.

In Figure 10 (a) (resp. Figure 10 (b)), we have represented the eco-neighborhood manager's cumulative utility at time period $T=20$ as a function of the error coefficient $\varepsilon$ under equal allocation (resp. priority based allocation) of the resource. We observe that in both cases, the increase of the error makes the eco-neighborhood manager's cumulative utility decrease and that storage is always preferable over no storage. Furthermore, storage is all the more interesting compared to no storage, as the error in the prediction increases.

\footnotetext{
${ }^{4}$ The choice of a quadratic cost function is rather arbitrary and the derived results can be extended to more general convex functions without loss of generalities, as mentioned in [9].

${ }^{5}$ It can be justified by the fact that depending on the exterior temperature, the houses' actions will be rather homogeneous i.e., maximum heat for low exterior temperature, minimal heat for high exterior temperature, etc., and by sheep of Panurge effect regarding storage.
} 


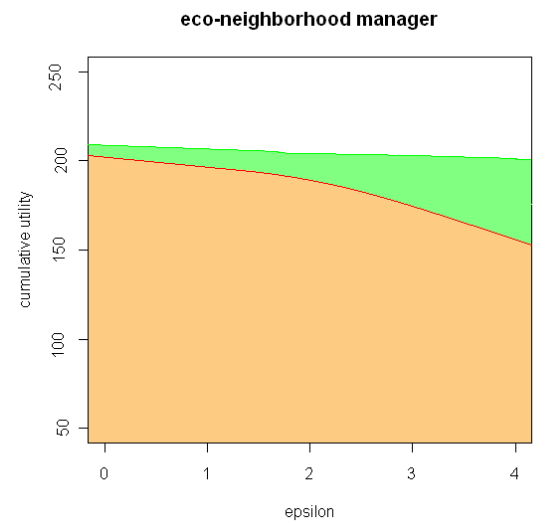

(a)

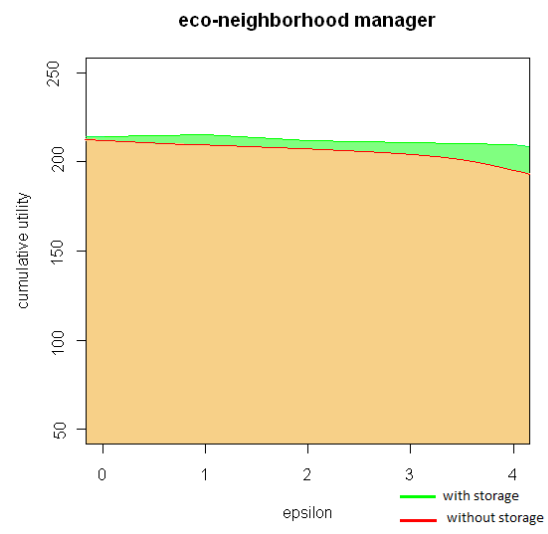

(b)

Figure 10: Eco-neighborhood manager's cumulative discounted utilities at $T=20$ under equal allocation scheme (a) and under priority allocation scheme (b) with dynamic pricing as a function of the error in forecasting $\varepsilon$.

\section{Conclusion}

We have modeled the problem of energy consumption planning in the eco-neighborhood, as a stochastic game and proposed algorithms based on stochastic dynamic programming to simulate its evolution under two resource allocation schemes: an equal allocation of the resource produced by the eco-neighborhood and a priority based one. The eco-neighborhood's energy policy has been simulated under various pricing mechanisms: flat rate, peak and off-peak hour, blue/white/red day, peak day clearing and a dynamic update of the price based on the eco-neighborhood's consumption. We observe that high incentives for house inhabitants to store energy is not straightforward since it relies heavily on the implemented pricing mechanism and on the homogeneity in the houses' characteristics.

A possible solution to promote storage in houses is to use dynamic pricing since under such a pricing mechanism, storage appears as a compensation for the errors made in the demand prediction when planning the eco-neighborhood's behavior and since in this case, storage generates a higher discounted cumulative weighted social welfare for the eco-neighborhood than no storage. Of course, dynamic pricing can be binding because it requires anticipating price changes and a high flexibility in task scheduling. Other pricing mechanisms based on contracts including various commitment lengths [2] or budget rebates [10] already launched by the telecommunications industry, can be envisaged. Besides, to be viable such approaches require investments in new production capabilities such as solar panels, wind farms, and also an extensive research and development effort to enhance the performance of storage capacity and the certification of demand response to avoid artifical retention at the end user level. These problem solving is necessary to the efficient deployment of the capacity market defined 
by the Nome law.

\section{References}

[1] Başar T., Olsder G. J., Dynamic Noncooperative Game Theory, SIAM Series in Classics in Applied Mathematics, 1999

[2] Bolton P., Dewatripoint M, Contract Theory, MIT Press, 2005

[3] Filar J., Vrieze K., Competitive Markov Decision Processes, Springer, 1996

[4] Häggström O., Finite Markov Chains and Algorithmic Applications, Cambridge University Press, 2002

[5] Kalagnanam J., Parkes D. C., Auctions, Bidding and Exchange Design, in Handbook of Quantitative Supply Chain Analysis: Modeling in the E-Business Era, D. Simchi-Levi, S. D. Wu, M. Shen (eds.), Chapter 5, Kluwer, 2004

[6] Kohlschein C., An Introduction to Hidden Markov Models. Probability and Randomization in Computer Science, Seminar in winter semester 2006/2007, Aachen university

[7] Kolokotsa D., Rovas D., Kosmatopoulos E., Kalaitzakis K., A roadmap towards intelligent net zero- and postive-energy buildings, Solar Energy, vol.85, pp.30653160,2011

[8] Le Cadre H., Bedo J.-S., Distributed Learning in Hierarchical Networks, in proc. of the 6-th International Conference on Performance Evaluation Methodologies and Tools, ValueTools 2012

[9] Le Cadre H., Bedo J.-S., Collaborative Learning is Better, working paper available at https://sites.google.com/site/helenelecadre, 2012

[10] Loiseau P., Schwartz G., Musacchio J., Amin S., Sastry S. S., Congestion Pricing Using a Raffle-Based Scheme, in proc. of Netgcoop 2011

[11] Maity I., Rao S., Simulation and pricing mechanism analysis of a solar-powered electrical microgrid, IEEE Systems Journal, vol.4, pp.275-284, 2012

[12] Mohsenian-Rad H., Wong V. W. S. , Jatskevich J., Schober R., Leon-Garcia A., Autonomous demand side management based on game-theoretic energy consumption scheduling for the future smart grid, IEEE Transactions on Smart Grid, vol.1, pp.320-331, 2010

[13] Myerson R., Game Theory: Analysis of Conflict, Harvard University Press, 2004

[14] Powell W. B., Approximate Dynamic Programming: Solving the Curses of Dimensionality, 2-nd Edition, Wiley Series in Probability and Statistics, 2007 
[15] Ross S., Introduction to Stochastic Dynamic Programming, Academic Press, 1983

[16] Saad W., Han Z., Poor V. H., Coalitional Game Theory for Cooperative MicroGrid Distribution Networks, 2-nd IEEE International Workshop on Smart Grid Communications, 2011

[17] Saad W., Han Z., Debbah M., Hjorrungnes A., Başar T., Coalition Game Theory for Communication Networks: A Tutorial, IEEE Signal Processing Magazine, Special Issue on Game Theory, vol.26, pp.77 - -97, 2009

[18] Saad W., Han Z., Poor H. V., Başar T., Game Theoretic Methods for the Smart Grid, IEEE Signal Processing Magazine, Special Issue on Signal Processing Techniques for the Smart Grid, 2012

[19] Showers M., Demand Response in the US Industrial Sector, Internal report of the Centre for Applied Mathematics, MINES ParisTech, 2012

[20] Spolakis E., Palyvos J. A., On the temperature dependence of photovoltaic module electrical performance: A review of efficiency/power correlations, Solar Energy, vol.83, pp.614-624, 2009

[21] Tijms H. C., A First Course in Stochastic Models, John Wiley \& Sons, 2003

[22] Voice T. D., Vytelingum P., Ramchurn S., Rogers A., Jennings N. R., Decentralised Control of Micro-Storage in the Smart Grid, in proc. 25-th Conference on Artificial Intelligence (AAAI 11)

[23] Vytelingum P., Voice T. D., Ramchurn S. D., Rogers A., Jennings N. R., Agentbased Micro-Storage Management for the Smart Grid, in proc. 9-th International Conferencee on Autonomous Agents and Multiagent Systems (AAMAS 2012)

[24] Young P., Strategic Learning and Its Limits, Oxford University Press, 2005

[25] From utopia to urban ecosystems, Alternatives magazine $n^{\circ} 22,2009$

[26] http://france.edf.com/ 